\title{
Preferencias sobre las urbanizaciones cerradas como modelo urbanístico en la ciudad de Varsovia*
}

\author{
Recibido: 2 de abril de 2020 - Aprobado: 21 de abril de 2020 \\ https://doi.org/10.22395/seec.v23n54a13
}

Magdalena Fuhrmann ${ }^{* *}$

\section{RESUMEN}

Las urbanizaciones cerradas fueron una de las formas de desarrollo de la vivienda en Polonia durante la última década del siglo XX e inicios del XXI. Las ciudades experimentaron una especie de florecimiento en este tipo de vivienda. Sin embargo, en la actualidad estas urbanizaciones cerradas han dejado de ser relevantes entre los planificadores urbanísticos, las autoridades de las ciudades, así como entre los residentes. El objetivo de este artículo es presentar los resultados derivados de una encuesta con una muestra de ciento una personas sobre las preferencias de vivienda en Varsovia. Se concluye que la falta de interés sobre los pisos en urbanizaciones cerradas responde a un asunto de percepción en el prestigio y avance socioeconómico de las personas.

\section{PALABRAS CLAVE}

Varsovia; urbanizaciones cerradas; preferencias de vivienda.

\section{CLASIFICACIÓN JEL}

P25

\section{CONTENIDO}

Introducción; 1. Desarrollo de la urbanización en Varsovia; 2. Ejemplos de urbanizaciones cerradas en Varsovia; 3. Preferencia de vivienda; ¿Es la urbanización cerrada lo que estamos buscando?; 4. Conclusiones; Referencias.

Artículo de investigación derivado de proyectos de tutorías de Magdalena Fuhrmann. Facultad de Geografía y Estudios Regionales de la Universidad de Varsovia, Polonia.

.. Doctora, Facultad de Geografía y Estudios Regionales, Universidad de Varsovia, Polonia. Correo electrónico: mfuhrmann@uw.edu.pl. Orcid: http://orcid.org/0000-0002-7015-6356 


\title{
Preferences on gated communities as a urban model in the city of Warsaw
}

\begin{abstract}
Gated communities were one of the ways in which housing developed in Poland during the last decade of the XXth century and the first one of the XXI. Cities experienced a kind of blooming on this type of housing; nonetheless, nowadays, these type of housing have become irrelevant for urban planners, city authorities and residents. The objective of this article is presenting the results derived from a poll with a total number of 101 participants on their housing preferences in Warsaw, We conclude that the lack of interest on flats in gated communities corresponds to a matter of perception of prestige and social and economical progress among people.
\end{abstract}

\section{KEYWORDS}

Warsaw; gated communities; home preferences.

\section{JEL CODE}

P25

\section{CONTENTS}

Introduction; 1. Development of urbanisation in Warsaw; 2. Examples of closed communities in Warsaw; 3. Housing preferences; Are closed communities what we are looking for?; 4. Conclusions; Bibliography.

\section{Preferências sobre as urbanizações fechadas como modelo urbanístico na cidade de Varsóvia}

\section{RESUMO}

As urbanizações fechadas foram uma das formas de desenvolvimento habitacional na Polônia durante a última década do século XX e início do XXI. As cidades experimentaram uma espécie de florescimento nesse tipo de residência; contudo, na atualidade, essas urbanizações fechadas vêm deixando de ser relevantes para os planejadores urbanísticos, para as autoridades das cidades e para os moradores. O objetivo deste artigo é apresentar os resultados derivados de uma pesquisa com uma amostra de 101 pessoas sobre as preferências de moradia em Varsóvia. Conclui-se que a falta de interesse sobre as propriedades em urbanizações fechadas responde a um assunto de percepção no prestígio e avanço socioeconômico das pessoas.

\section{PALAVRAS-CHAVE}

Varsóvia; urbanizações fechadas; preferências de moradia.

\section{CLASSIFICAÇÃO JEL}

\author{
P25
}

\section{CONTEÚDO}

Introdução; 1. Desenvolvimento da urbanização em Varsóvia; 2. Exemplos de urbanizações fechadas em Varsóvia; 3. Preferência de moradia; O que estamos procurando é a urbanização fechada?; 4. Conclusões; Referências. 


\section{INTRODUCCIÓN}

Las urbanizaciones cerradas son

unidades residenciales amuralladas o cercadas a las que se restringe el acceso público, a menudo custodiadas mediante el uso de tecnologías de la información y las comunicaciones o personal especial, y normalmente reguladas por documentos que obligan a los residentes a tener normas especificas de convivencia vecinal (Owczarek, 2011, citado por Blandy et al., 2003).

En lo que respecta al desarrollo de la vivienda, no solo se utiliza el término de urbanización cerrada, sino de cercada y vigilada (Owczarek, 2011). Esta distinción indica el uso de diferentes elementos de protección: una pared física y una empresa de seguridad y vigilancia por video.

Las urbanizaciones cerradas comenzaron a construirse originalmente en los Estados Unidos. Se hicieron en tres variedades (Blakely y Snyder, 1997a):

- lifestyle community

- elite community

- security zones

Se diferenciaban en la forma de desarrollo, la organización especial y la composición social. Las primeras (lifestyle community: comunidad de estilo de vida) son urbanizaciones que reúnen a ancianos, jubilados y personas que juegan al golf. El establecimiento de barrios comunitarios de elite se asocia con la necesidad de destacar el prestigio y la posición financiera. El último tipo de urbanizaciones cerradas indicadas por Blakely y Snyder (1997b) son zonas de seguridad situadas en barrios pobres de los suburbios.

Entre los factores que influyen en el establecimiento de urbanizaciones cerradas figuran el deseo de aumentar la sensación de seguridad (independientemente del nivel real de delincuencia), los factores culturales y las tendencias nacionales, pero también la necesidad de copiar las pautas occidentales (especialmente en los países de Europa central), así como el deseo de estar entre personas similares (Kłopotowska, 2010; Morawska, 2018). Según Glasze, Webster y Frantz (2006), las urbanizaciones cerradas son un producto del mercado de la vivienda (mercado inmobiliario), que responde a la necesidad de seguridad y prestigio.

\section{DESARROLLO DE LA URBANIZACIÓN EN VARSOVIA}

El desarrollo residencial de Varsovia es muy diverso. En general, hay edificios del periodo anterior a la guerra (antes de 1939), edificios de 1945 a 1989 y edificios 
construidos después de 1989. Sin embargo, esta última también es bastante diversa. Los edificios residenciales más antiguos de Varsovia son casas de vecindad, situadas principalmente en los distritos centrales de la ciudad. Los edificios de los años 1945-1989 son en mayoría viviendas multifamiliares, construidas con métodos prefabricados. El establecimiento de urbanizaciones en este periodo era responsabilidad del Estado y de las cooperativas de viviendas, y todo el proceso de construcción estaba completamente centralizado.

Después de 1989, es decir, durante el periodo de transformación, el mercado de la vivienda adoptó los principios de libre mercado. Por ello se descentralizaron las construcciones de nuevas urbanizaciones y se incorporaron al proceso inversores privados. Un rasgo característico de las nuevas urbanizaciones construidas en los años noventa es el hecho de que fueron construidas con una diversidad arquitectónica y estética: los edificios son a menudo coloridos, pero no se ajustan al entorno.

A principios del siglo XXI, la calidad de la arquitectura de las viviendas mejoró. Las clásicas urbanizaciones multifamiliares se seguían construyendo, pero también aparecieron las urbanizaciones cerradas. Estas ganaron rápidamente sus partidarios, y el principal factor que creó tanto la demanda como la oferta de estas fincas fue la seguridad y la sensación de seguridad ampliamente entendidas. Cercar la finca era garantizar la seguridad de los residentes. Se suponía que los separaría de los demás, de la gente y del espacio. A lo largo de los años, las opiniones sobre las urbanizaciones cerradas han cambiado. Inicialmente, fueron tratadas como un oasis de riqueza o lujo, una especie de gueto en la ciudad. Más tarde resultó que los residentes no se sentían asegurados en estos barrios de viviendas, y el mismo hecho de estar separados del espacio de la ciudad comenzó a perturbarlos. Los expertos también indicaron el impacto negativo de las urbanizaciones cerradas en el espacio urbano, que no son partidarios de cercar la ciudad, apagar barrios enteros ni de la apropiación del espacio.

También es necesario explicar cómo se construyeron las nuevas urbanizaciones después de 1989 (y hasta ahora). La construcción de urbanizaciones, es decir, la entrega de pisos en el mercado es responsabilidad de entidades públicas (autoridades estatales, autoridades locales) y otras entidades (cooperativas de viviendas y empresas privadas). Las viviendas construidas por entidades estatales son instalaciones para personas de bajos ingresos que no pueden permitirse comprar un piso. Por otro lado, las empresas privadas proporcionan apartamentos a las personas que los compran en efectivo o a crédito bancario. La cuestión de las urbanizaciones cerradas se refiere en realidad a las urbanizaciones establecidas por empresas privadas o cooperativas de viviendas (un tipo específico de empresa comercial). 
Los pisos de la propiedad de entidades públicas están situados en las partes antiguas de las ciudades (los edificios residenciales más antiguos) y los edificios nuevos no se construyen a gran escala y son en realidad edificios individuales, no barrios de viviendas.

¿Cuáles son las limitaciones de la empresa privada para construir una nueva urbanización? Estas restricciones son el resultado de los reglamentos de zonificación. Hay dos situaciones: cuando una empresa inmobiliaria construye en un lugar para el que se adopta un plan local (plan urbanístico) y cuando no existe dicho plan. En el primer caso, la empresa está obligada a cumplir las disposiciones del plan local, pero estas se refieren principalmente a la altura del desarrollo, la coloración de la elevación, la proporción de la elevación, la proporción de zonas abiertas y la finalidad del edificio. No hay registros de cercado de la finca. La solución a esta situación, en la que las empresas pueden realmente construir "como quieran", fue un proyecto de resolución paisajística preparado en Varsovia, según el cual no se podían construir nuevas fincas valladas en Varsovia. El reglamento prohibía cercar zonas de más de dos hectáreas. Lamentablemente, esta resolución fue rechazada por las autoridades provinciales y no entró en vigor. Además, en Varsovia -pero no solo allí- existe un fenómeno de cercado "secundario", es decir, el cercado de urbanización ya existente, a menudo construido antes de 1989.

\section{EJEMPLOS DE URBANIZACIONES CERRADAS EN VARSOVIA}

Las primeras urbanizaciones cerradas en Varsovia se construyeron a finales de los años noventa del siglo XX. El desarrollo de los asentamientos cercados en Varsovia cae a principios del siglo XXI (Gądecki, 2009). Según Lewicka y Zaborska (2007), más de cuatrocientas urbanizaciones de ese tipo se encontraban entonces en Varsovia. Las urbanizaciones cerradas en Varsovia se construyeron (y se construyen) en prácticamente todos los distritos, pero difieren en:

- La superficie.

- La oferta.

- El desarrollo de los espacios comunes.

- La diversidad del desarrollo.

- El grado de cierre real.

- El grado de complimiento de las normas de acceso a la urbanización para las personas de fuera. 
Sobre todo, se deben distinguir las grandes urbanizaciones cerradas, que pueden llamarse "ciudades en la ciudad", porque detrás de la muralla no solo hay bloques de pisos, sino también tiendas y puntos de servicio para atender a los residentes. Sin embargo, lo más frecuente era que en Varsovia se construyeran las urbanizaciones cerradas, que detrás de la muralla solo incluían edificios residenciales, aparcamientos o zonas de juego para niños. Todas las tiendas y puntos de servicio se encuentran fuera de la urbanización. Un ejemplo de una gran urbanización cerrada es Marina Mokotów, una urbanización con una superficie de $186.160 \mathrm{~m}^{2}$. La urbanización se ha desarrollado en una extensa e intensiva urbanización multifamiliar. Los edificios más altos tienen siete pisos. Las calles atraviesan el barrio residencial, formando rutas de comunicación (gráfico 1).

\section{Gráfico 1. Marina Mokotów. La gran urbanización cerrada en Varsovia}

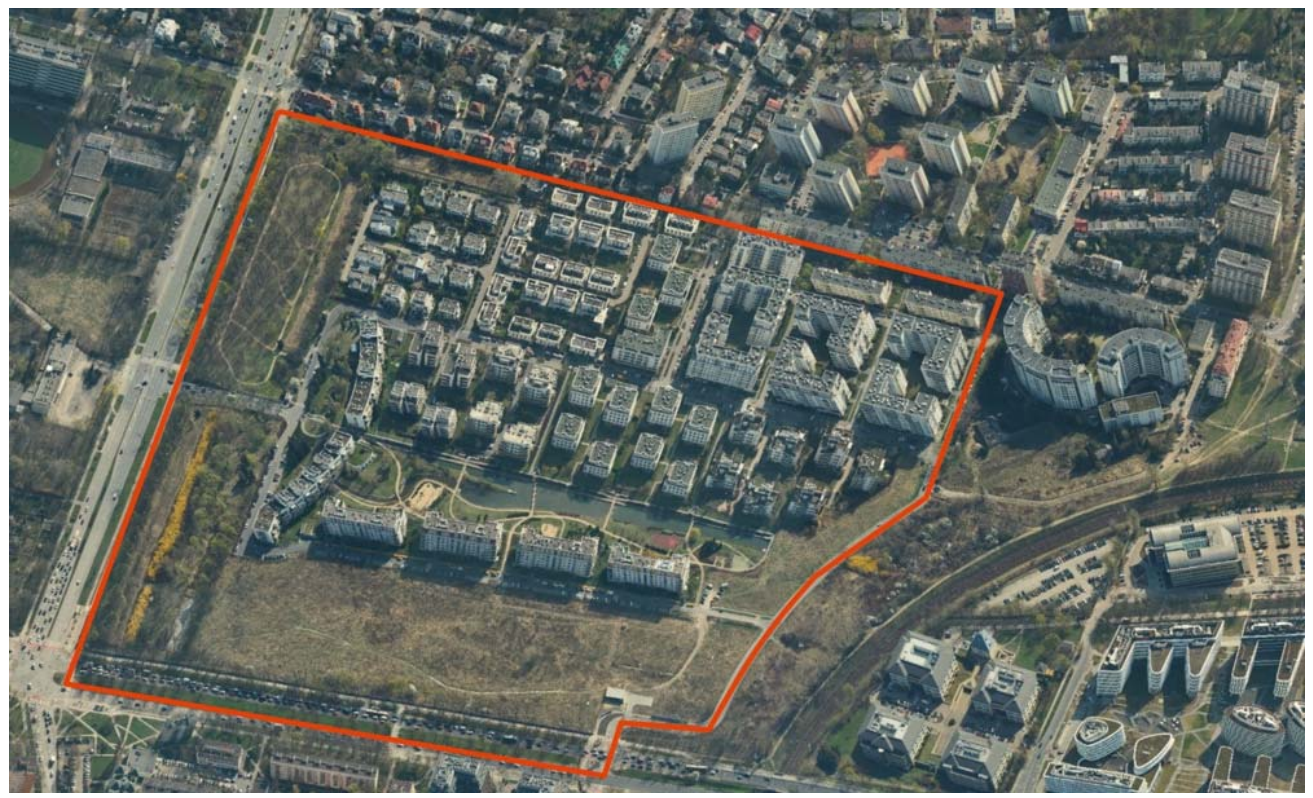

Fuente: Ukosne (2020).

Un ejemplo de pequeñas urbanizaciones cerradas, es decir, las que no ofrecen tiendas u otros servicios "detrás de la pared", es la urbanización Derby en Białołęka -en realidad una colección de muchas pequeñas urbanizaciones cerradas construidas por una empresa-, cada una de ellas está cercada y protegida (gráfico 2). Este es un ejemplo de la separación de barrios más pequeños, separados entre sí por vallas y senderos. No hay espacios comunes planeados aquí. 
Preferencias sobre las urbanizaciones cerradas como modelo urbanístico en la ciudad de Varsovia

\section{Gráfico 2. Urbanización Derby en Varsovia}

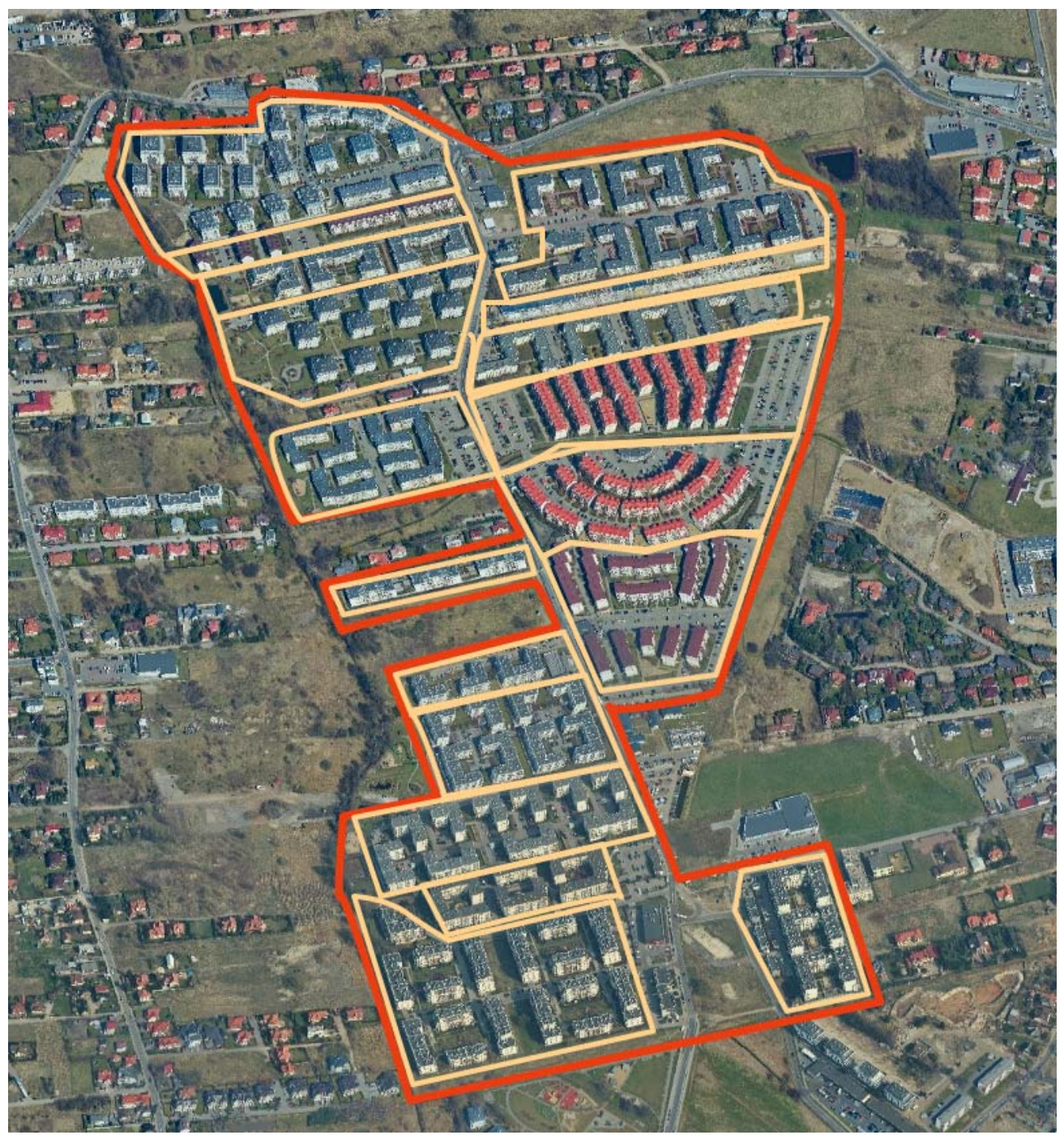

Fuente: Ukosne (2020).

Otro ejemplo de pequeñas urbanizaciones cercadas son las situadas en Mokotów, que forman parte de complejo Eko-Park (gráfico 3). En este caso, el complejo también consta de varias estructuras cercadas más pequeñas. Sin embargo, la valla está sujeta a un pequeño espacio que ya no incluye aparcamientos y zonas de juego (como en el caso de Derby), sino que los espacios comunes (tiendas, aparcamientos y espacio de recreo) están situados fuera de la valla. 


\section{Gráfico 3. Eko-Park urbanización en Mokotów}

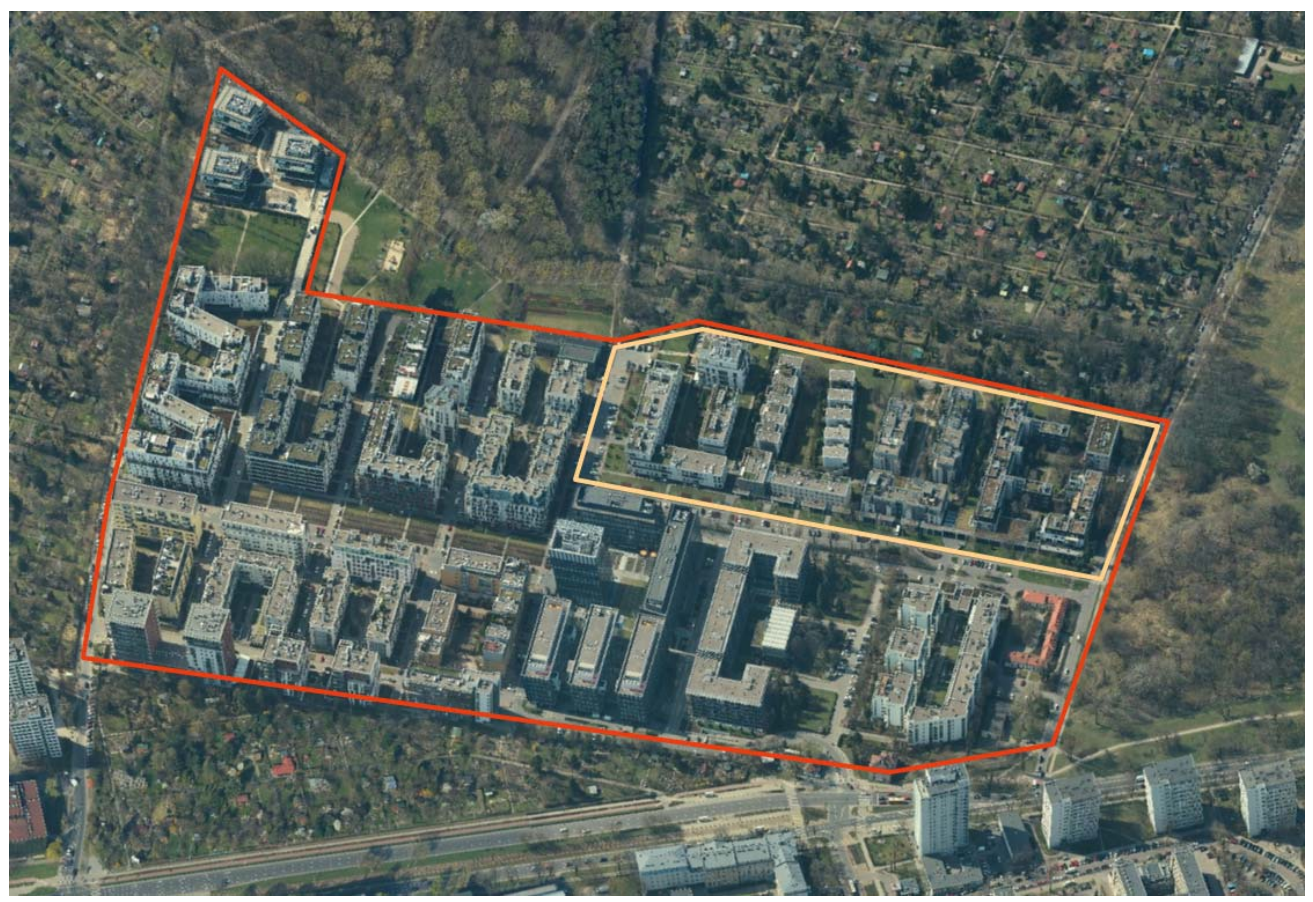

Fuente: Ukosne (2020).

Los dos ejemplos anteriores -Derby y Eko-Park- muestran un enfoque diferente de las urbanizaciones cercadas. Se trata de fincas situadas en dos distritos diferentes de la ciudad (Białołęka en el noreste) y Mokotów (parte central de Varsovia), lo que se traduce directamente en el precio de los apartamentos. Sin embargo, estas propiedades difieren en el estándar. La urbanización de Mokotów se construyó con mejores materiales y el diseño arquitectónico es más avanzado. La urbanización de Derby ha sido construida desde 2003, pero la última parte de ella se puso en funcionamiento en 2019. Eko-Park es una urbanización construida desde 2008. Otras diferencias entre estos asentamientos se refieren a la organización del espacio fuera de las vallas. En el caso de Eko-Park, no solo posee tiendas, sino que los cafés y restaurantes se han ubicado en este espacio, lo que fomenta la integración de los habitantes y les permite ir mas allá de la manzana. En el caso de la finca de Derby, este tipo de oferta es mucho más modesta, a pesar del mayor número de residentes. Solo tienen a su disposición las tiendas de suministros diarios. 
Otro tipo de urbanización con vallas es la finca Arbuzowa, también en Mokotów, que es otro ejemplo de una urbanización con vallas (gráfico 4.). La urbanización fue fundada en1987 y es una urbanización prefabricada. Actualmente, es inaccesible para las personas de fuera. Todas las entradas a la urbanización están valladas (barreras) y los lugares restantes están rodeados por un seto. Esto limita la entrada de coches, pero no es un gran obstáculo para peatones.

\section{Gráfico 4. Arbuzowa. Urbanización en Mokotów}

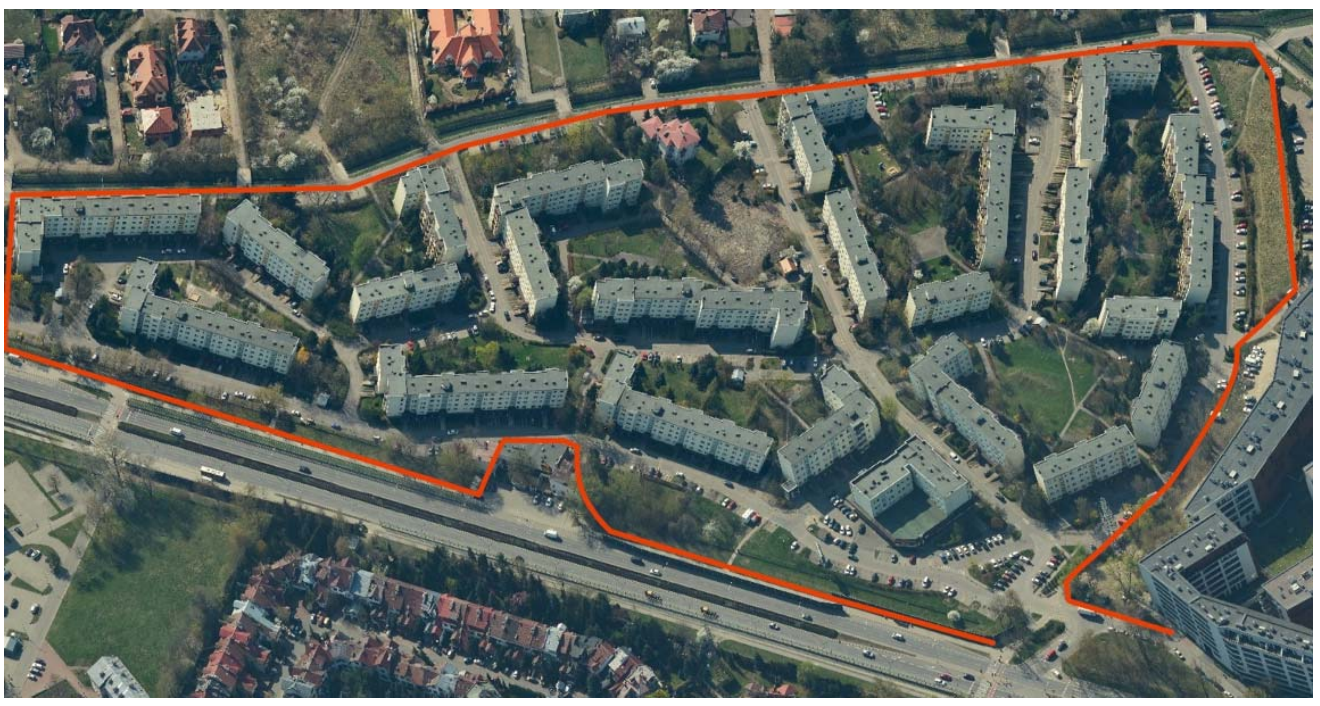

Fuente: Ukosne (2020).

\section{PREFERENCIA DE VIVIENDA ¿ES LA URBANIZACIÓN CERRADA LO QUE ESTAMOS BUSCANDO?}

A fin de comprobar si las urbanizaciones cerradas siguen siendo elegidas frecuentemente como lugar de residencia, se realizó una encuesta sobe las preferencias de vivienda'. En la encuesta participaron personas que, en el momento de la participación en la encuesta, querían comprar o compraron un piso en Varsovia en los tres meses anteriores a la encuesta. En el estudio preliminar, cuyos resultados se presentarán en este articulo, participaron ciento un encuestados. La encuesta se llevó a cabo a través de foros de internet que reunían a personas que buscaban un piso en Varsovia.

La investigación realizada como parte de la tesis de licenciatura de T. Wagemans en la Facultad de Geografía y Estudios Regionales de la Universidad de Varsovia. La tutora: Magdalena Fuhrmann. 
La mayoría de los encuestados eran mujeres (que constituían el 73,3\% de todos los encuestados) y personas con educación superior (60,4\%). Las personas encuestadas procedían en su mayoría de Varsovia (40,6 \%), pero una parte relativamente grande de las personas procedía de otras ciudades del Voivodía y otras localidades, de otras ciudades y zonas rurales (gráfico 5). También se muestra que Varsovia es un mercado residencial atractivo y atrae a nuevos residentes. La edad media de los encuestados es de 25,04 años (desviación estándar 5,553). Según un informe de Metrohouse y Gold Finance de 2019, la mayoría de las personas que compraron apartamientos en Varsovia tenían hasta cuarenta años. Por consiguiente, la estructura de edad de los encuestados es en gran medida similar a las tendencias observadas.

\section{Gráfico 5. Lugar de nacimiento de los encuestados}

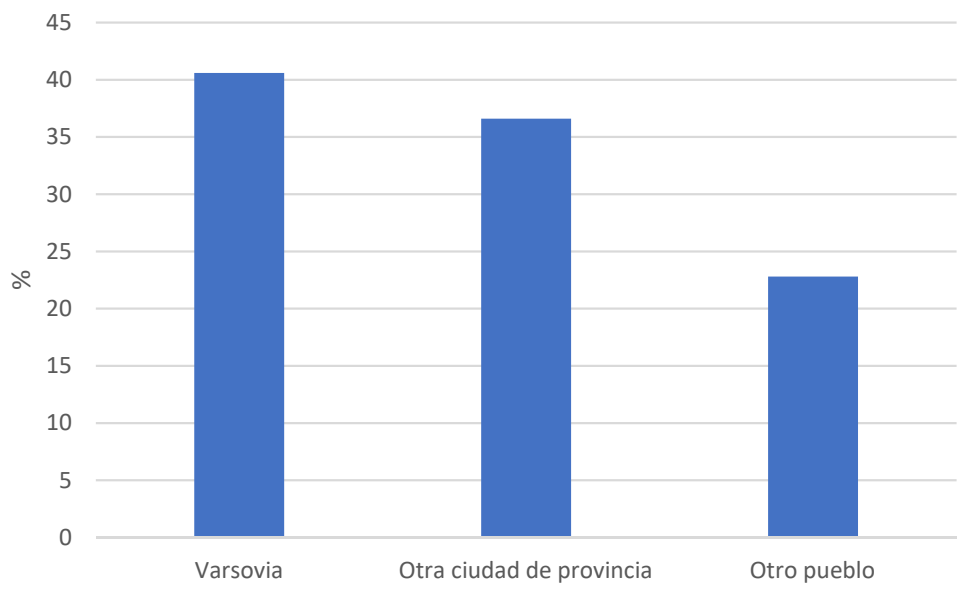

Fuente: elaboración propia basado en la investigación de Wagemans (2018).

La encuesta realizada tuvo en cuenta las características de las preferencias de vivienda, incluidas las urbanizaciones cerradas. Las personas que actualmente buscan un piso en Varsovia no solían vivar antes y no viven actualmente en una urbanización cerrada (58,4\%). Así que estas son personas que no tienen ninguna experiencia de vivir en este tipo de viviendas. El resto de los encuestados tienen esa experiencia, pero 12,9\% de los encuestados no viven actualmente en un complejo residencial cerrado (gráfico 6). 
Gráfico 6. La experiencia de vivir en una urbanización cerrada

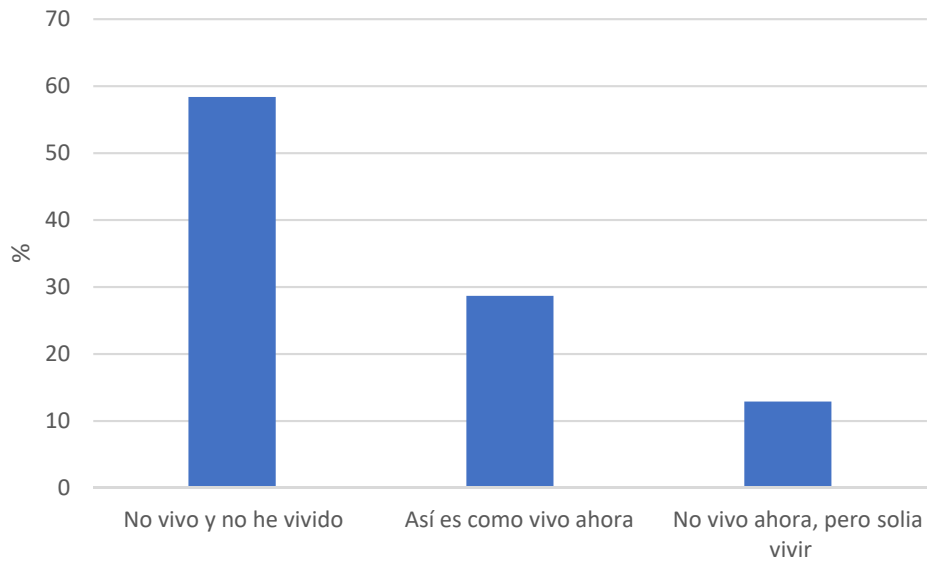

Fuente: elaboración propia basado en la investigación de Wagemans (2018).

\section{Gráfico 7. Interés en vivir en una urbanización cerrada}

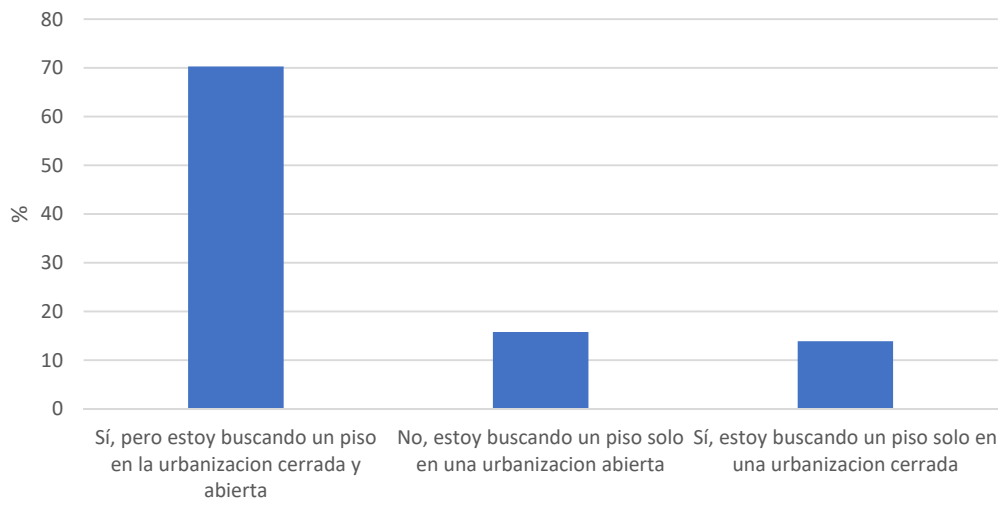

Fuente: propio estudio basado en la investigación de Wagemans (2018).

La mayoría de los encuestados buscan un piso tanto en urbanizaciones "abiertas" como en urbanizaciones cerradas (70,3 \%), casi el 14 \% de los encuestados están interesados solo en pisos en el terreno de fincas cerradas. Así que se puede ver que el interés no es muy amplio. Para los que tienen en cuenta la compra de un piso en una urbanización cerrada, el hecho de vallar es importante en diversos grados: para $40 \%$ de los encuestados tiene poca importancia, pero para 35,3\% es una característica muy importante del piso comprado (gráfico 7). Tal vez la voluntad de comprar un apartamento en una urbanización cerrada no este directamente 
relacionada con el hecho de vallar (cuestiones de sensación de seguridad), sino más bien con el sentimiento de prestigio. Hay que destacar que las fincas cerradas en Varsovia se asociaban a un nivel más alto, de mayor clase y prestigio.

El hecho de que los asentamientos cercados en Varsovia se estén construyendo también se ve muy diferente. Casi el $35 \%$ de los encuestados considera que esta dirección de desarrollo residencial es negativa y el 45,7 \% la considera muy positiva. Esto demuestra que los encuestados perciben la tendencia y sus preferencias de manera diferente. Hay pocas personas que estén decididas a comprar un piso en una urbanización cerrada, mientras que hay más valoraciones positivas para la propia tendencia. Esto significa que el fenómeno mismo de tales asentamientos no es malo, pero no es lo que los encuestados esperan.

Los encuestados son coherentes en sus opiniones, lo que se refleja en el tipo de forma de vivienda preferida (tabla 1). Se identificó una relación entre las experiencias de los encuestados con los asentamientos cerrados y sus búsquedas actuales $\left(\mathrm{Chi}^{2}=12,011 ; d f=4 ; p=0,017\right)$. Las personas que no tienen ninguna experiencia de vivir en una urbanización cerrada son ahora mucho más propensas a buscar un piso en una urbanización abierta ( $81,2 \%$ ). Al mismo tiempo, el porcentaje de personas que actualmente buscan un piso en una urbanización cerrada es el más alto entre los dos tipos de urbanizaciones (57,1\%).

La investigación también permitió indicar la tendencia estadística relativa a la relación entre la experiencia de los encuestados y su evaluación de la tendencia a construir asentamientos cerrados ( $\left.C h i^{2}=8,947 ; d f=4 ; p=0,062\right)$. Entre las personas que evalúan esta tendencia negativamente, el porcentaje más alto es el de las que no tienen experiencia en vivir en una urbanización de este tipo (tabla 2). Este es un ejemplo de evaluación negativa, un fenómeno que el encuestado no conoce. Al mismo tiempo, cabe señalar que entre quienes evalúan positivamente esta tendencia, también estas personas tienen el porcentaje más alto, pero es del 54,8 \%. El mayor porcentaje de personas que viven actualmente en una urbanización cerrada se identificó los que evalúan positivamente la tendencia.

Las personas que actualmente buscan un piso, tanto en urbanizaciones abiertas como cerradas, suelen dar una positiva evaluación de la tendencia de la construcción de urbanizaciones cerradas (tabla 3). El estudio también mostró un vínculo entre estas características (Chi $\left.{ }^{2}=26,488 ; d f=4 ; p<0,0001\right)$. Por consiguiente, las personas que tienen una opinión negativa de la tendencia a la construcción de urbanizaciones cerradas no solo están interesadas en esos pisos, sino que también consideran la posibilidad de elegir un piso en una urbanización de ese tipo. 
Las preferencias de los encuestados también mostraron una conexión con la educación $\left(\mathrm{Chi}^{2}=6,879\right.$; $\left.\mathrm{df}=2 ; p<0,032\right)$. Se observó un mayor porcentaje de personas que se interesan por las urbanizaciones cerradas solo entre las personas con educación superior (18,0 \%) que entre las personas con educación secundaria (7,5\%). Aquí también, se puede suponer que puede tener que ver con un cierto sentido de prestigio y también con la riqueza. No se demostró ninguna conexión entre las preferencias de vivienda y la evaluación de tendencia a construir urbanizaciones cerradas y le lugar de nacimiento (origen) de los encuestados.

Tabla 1. Relación entre la experiencia y el tipo de alojamiento preferido

\begin{tabular}{|c|c|c|c|c|}
\hline \multirow{5}{*}{ 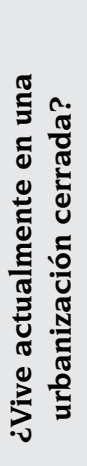 } & & \multicolumn{3}{|c|}{ ¿Está considerando comprar un piso en una urbanización cerrada? } \\
\hline & & $\begin{array}{l}\text { No, solo en una urbani- } \\
\text { zación abierta }\end{array}$ & $\begin{array}{c}\text { Sí, pero en dos tipos de } \\
\text { urbanizaciones }\end{array}$ & $\begin{array}{l}\text { Sí, solo en una urbani- } \\
\text { zación cerrada }\end{array}$ \\
\hline & $\begin{array}{l}\text { No, pero he } \\
\text { vivido antes }\end{array}$ & $18,8 \%$ & $12,7 \%$ & $7,1 \%$ \\
\hline & $\begin{array}{l}\text { No y no he } \\
\text { vivido antes }\end{array}$ & $81,2 \%$ & $57,7 \%$ & $35,7 \%$ \\
\hline & $\begin{array}{l}\text { Sí, vivo } \\
\text { actualmente }\end{array}$ & $0,0 \%$ & $29,6 \%$ & $57,1 \%$ \\
\hline & & $100,0 \%$ & $100,0 \%$ & $100,0 \%$ \\
\hline
\end{tabular}

Fuente: elaboración propia basada en la investigación de Wagemans (2018).

Tabla 2. Relación entre la experiencia y la evaluación de la tendencia a construir urbanizaciones cerradas

\begin{tabular}{|c|c|c|c|c|}
\hline \multirow{6}{*}{ 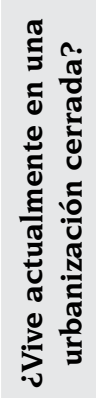 } & & \multicolumn{3}{|c|}{$\begin{array}{c}\text { ¿Cómo evalúa la tendencia a construir } \\
\text { urbanizaciones cerradas? }\end{array}$} \\
\hline & & Mal & Medio & Bien \\
\hline & No, pero he vivido antes & $12,5 \%$ & $22,2 \%$ & $7,1 \%$ \\
\hline & No y no he vivido antes & $75,0 \%$ & $44,4 \%$ & $54,8 \%$ \\
\hline & Sí, vivo actualmente & $12,5 \%$ & $33,3 \%$ & $38,1 \%$ \\
\hline & & $100,0 \%$ & $100,0 \%$ & $100,0 \%$ \\
\hline
\end{tabular}

Fuente: elaboración propia basada en la investigación de Wagemans (2018). 
Tabla 3. Relación entre las preferencias de los encuestados y la evaluación e la tendencia a construir urbanizaciones cerradas

\begin{tabular}{|c|c|c|c|c|}
\hline \multirow{5}{*}{ 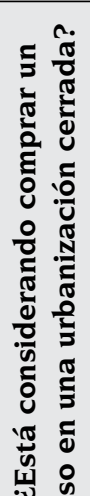 } & & \multicolumn{3}{|c|}{ ¿Cómo evalúa la tendencia a construir urbanizaciones cerradas? } \\
\hline & & Mal & Medio & Bien \\
\hline & $\begin{array}{l}\text { No, solo en } \\
\text { una urbaniza- } \\
\text { ción abierta }\end{array}$ & $37,5 \%$ & $11,1 \%$ & $2,4 \%$ \\
\hline & $\begin{array}{c}\text { Sí, pero en } \\
\text { dos tipos de } \\
\text { urbanizaciones }\end{array}$ & $62,5 \%$ & $83,3 \%$ & $69,0 \%$ \\
\hline & $\begin{array}{c}\text { Sí, solo en una } \\
\text { urbanización } \\
\text { cerrada }\end{array}$ & $0,0 \%$ & $5,6 \%$ & $28,6 \%$ \\
\hline & & $100,0 \%$ & $100,0 \%$ & $100,0 \%$ \\
\hline
\end{tabular}

Fuente: elaboración propia basada en la investigación de Wagemans (2018).

\section{CONCLUSIONES}

La investigación realizada mostró un interés bastante bajo en apartamentos en urbanizaciones cerradas. Los encuestados que decidieron vivir solo en ese lugar constituyeron únicamente el $4 \%$ de todos los encuestados. Hay un grupo de personas que no rechazan esta posibilidad, pero hay más gente que quiere vivir en una urbanización tradicional (15,8 \%). Del mismo modo, el hecho de vallar una urbanización no es un factor importante que se tenga en cuenta a la hora de buscar un piso. Hay que recordar que la selección de una urbanización cerrada no tiene por qué estar estrechamente relacionada con las cuestiones de seguridad propiamente dichas, sino también con el sentido del prestigio o el avance social.

¿Responden los resultados obtenidos a la pregunta sobre la escala del fenómeno? Hasta cierto punto, sí. Las investigaciones realizadas por otros autores en años anteriores muestran que el interés por las urbanizaciones cerradas y la aceptación de este tipo de desarrollo de viviendas fue mucho mayor. Según Jałowiecki, Krajewska y Olejniczak (2004), el 66 \% de los encuestados consideraba que los asentamientos cercados eran un fenómeno normal, solo el 20 \% calificaba este tipo de asentamientos como negativos y solo el 10 \% no quería que se construyeran. Según un estudio realizado en 2002 por el Royal Institute of Chartered Surveyors de 2002 (Blandy et al., 2003), más del $50 \%$ de los residentes británicos evaluaron positivamente urbanizaciones cercadas. En las encuestas de Lewicka y Zaborska (2007), más de 
la mitad de los encuestados eligieron como lugar de residencia las urbanizaciones cerradas, y la actitud ante el cercado fue positiva.

La investigación presentada en este artículo muestra una aceptación mucho menor para el cercado de urbanizaciones a partir de 2018; lo que puede indicar un cambio en el enfoque del tema. Las cuestiones presentadas requieren una labor e investigación adicional que ya ha emprendido el autor del artículo. En primer lugar, la tesis del bajo interés en las urbanizaciones cerradas se comprobará entre las empresas y en las oficinas de venta de pisos del mercado primario y secundario. La investigación presentada en este artículo también continuará. Una cierta premisa para decir que las urbanizaciones cerradas pueden no ser lo que más desean los residentes, puede ser el hecho de que los residentes de la mayor urbanización cerrada de Varsovia -Marina Mokotów- están considerando derribar los muros.

\section{BIBLIOGRAFÍA}

Blandy, Sarah; Lister, Diane; Atkinson, Rowland y Flint, John (2003). Gated Communities: A Systematic Review of the Research Experience. Bristol: ERSC Centre for Neighborhood Research, 45p.

Blakely, Edward y Snyder, Mary Gail (1997 a). Divided We Fall: Gated and Walled Communities in the United States. En: Ellin, Nan (Ed.). Architecture of Fear. Nueva York: Princeton Architectural Press, p. $\overline{85-100 .}$

Blakely, Edward y Snyder, Mary Gail (1997 b). Fortress America: Gated Communities in the United States. Washington: Brookings Institution Press, 208p.

Gądecki, Jacek (2009). Za murami. Osiedla grodzone w Polsce - analiza dyskursu. Breslavia: Wydawnictwo Uniwersytetu Wrocławskiego, 300 p.

Glasze, Georg; Webster, Chris y Frantz, Klaus (2006). Private cities. Global and local perspectives. Londres: Routledge, 256 p.

Jałowiecki, Bohdan; Krajewska, Magdalena y Olejniczak, Karol (2004). Klasa metropolitalna w przestrzeni Warszawy. En: Grzelaka, Janusza y Zaryckiego Tomasza (Eds.). Społeczna mapa Warszawy. Interdyscyplinarne studium metropolii warszawskiej. Varsovia: Wydawnictwo Naukowe, p. 132-146.

Kłopotowska Agnieszka (2010). Czynnik obronności we współczesnej architekturze zespołów mieszkalnych. En: Budownictwo i Architektura, vol. 6, n. ${ }^{\circ} 1,51-61$.

Lewicka, Maria y Zaborska, Katarzyna (2007). Osiedla zamknięte - czy istnieje alternatywa. En: Kolokwia Psychologiczne, n. ${ }^{\circ}$ 16, p. 135-152.

Morawska, Ilona (2018). Gettoizacja przestrzeni, czyli osiedla zamknięte w strukturze miast Polski i zagranicy. En: Człowiek i Środowisko, vol. 41, n. ${ }^{\circ}$, p. 95-112. 
Owczarek, Dominik (2011). Zamknięte osiedla, czyli dylemat współczesnych polskich miast. Badanie porównawcze mieszkańców zamkniętych i otwartych osiedli w Warszawie. En: Przegląd Socjologiczny, vol. 60, p. 365-391.

Ukosne (2020). Warszawa Ukosna. Varsovia: Polonia.

Wagemans, Tristan (2018). Perception of gated communities on the example of the capital city of Warsaw. http://wgsr.uw.edu.pl/wgsr/index.php/pl/strona-glowna-2-2/ 